\title{
Fast events detection with the CTS diagnostic on FTU and plans for improvement
}

\author{
A. Bruschi ${ }^{1}$, E. Alessi ${ }^{1}$, B. Baiocchi ${ }^{1}$, W. Bin ${ }^{1}$, O. D'Arcangelo ${ }^{2}$, F. Fanale ${ }^{1}$, L. Figini ${ }^{1}$, C. Galperti ${ }^{3}$, \\ S. Garavaglia ${ }^{1}$, G. Gittini ${ }^{1}$, G. Granucci ${ }^{1}$, G. Grosso ${ }^{1}$, L. Lubyako ${ }^{4}$, C. Mazzotta ${ }^{2}$, V. Mellera ${ }^{1}$, A. Moro ${ }^{1}$,

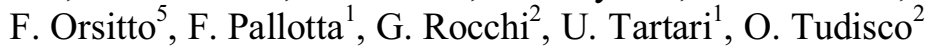 \\ ${ }^{1}$ Istituto di Fisica del Plasma - Consiglio Nazionale delle Ricerche, Milano, Italy, bruschi@ifp.cnr.it \\ ${ }^{2}$ ENEA C.R. Frascati, via E. Fermi 45, 00044 Frascati (Roma), Italy \\ ${ }^{3}$ Swiss Plasma Center, Ecole Polytéchnique Fédérale de Lausanne, Lausanne, Switzerland \\ ${ }^{4}$ Institute of Applied Physics, 46 Ulyanov st., Nizhny Novgorod, 603950, Russia \\ ${ }^{5}$ Consorzio CREATE, Università degli Studi di Napoli, Napoli, Italy
}

The capability of the CTS diagnostic to detect emissions at frequencies close to the injected EC power can be used to study the occurrence of parametric decay instabilities (PDI) excited by the ECH power. In this way, from the detection of back-scattered power in the case of 2 nd harmonic X-mode injection $[1,2]$, the sources of the signals were well explained by waves generated by PDI mechanisms [3]. On FTU, during the work with the CTS diagnostics focused on studying the effects of possible PDI occurrence on EC beams (under Enabling Research project ER-ENEA-06), other still unexplained fast signals were detected in the spectra, in experiments with injection of EC power in O-mode at the fundamental EC harmonic [4]. The possibility to analyze data with a high time resolution is crucial for the study of these fast events, and the use of a fast rate (12 GS/s max) digitizer and of the FFT of the sampled intermediate frequency signal (taken at the output of the $140 \mathrm{GHz}$ heterodyne radiometer front-end) over different time-scales is mandatory [5]. In this paper the observations obtained with new experiments are compared to the previous ones. The design of the new receiving line equipped with a RS antenna and the status of the work are presented.

\section{Experiment preparation}

On the diagnostic side an effort is ongoing to exclude any parasitic effect of gyrotron spectrum, of highfrequency front-end components, of local breakdown in the antenna and other disturbances. The gyrotron frequency spectrum, previously obtained from a lossy pickup line $50 \mathrm{~m}$ long used to measure the gyrotron main line frequency vs time (without using the notch filter), was now obtained with a direct pick-up from the stray radiation in the launcher region while shooting the gyrotron on the local dummy load. In this way a higher signal is collected and a sensitive spectrum of the gyrotron radiation is obtained, using the notch filter to suppress the main line. The resulting spectra from the gyrotron often include spurious lines that appear also in some CTS spectra from plasma, as shown in Fig. 1. The lines of this type have always the same behavior, starting from the beginning of the gyrotron pulse and lasting tens or hundreds of ms with a frequency decreasing with oscillations around an average speed of $2 \mathrm{GHz} / \mathrm{s}$ (Fig. 1 top right).

Whether these are due to gyrotron spurious mode or to a signal treatment in the HF diagnostic section is under evaluation. The level of the spurious line, not observed in the 2015 shots, is estimated at least $50 \mathrm{~dB}$ below the main gyrotron line.
The calibration of spectra received from the plasma is now performed comparing the spectra with the ones obtained during Ohmic pulses with the EC resonance inside the plasma (where the emission is only thermal ECE) and subtracting the noise spectra obtained during the same shot with the aid of a chopper/modulator working at around $10 \mathrm{kHz}$. The spectra obtained during the two chopping phases (on-off) are evaluated by proper data analysis, summed separately and then the noise part is subtracted. Examples of such spectra are shown in Fig. 2. Local breakdown in the antenna was monitored recording the light and the stray radiation level received at the back of the launching/receiving antenna, using a remote photomultiplier connected to an optical fiber as light detector and a mm-wave detector installed on a horn antenna looking into the port 8 , from where the probe is launched.

\section{Analysis of experimental observations}

The analysis work has been focused on the correlation of spectral features with other plasma diagnostic observations. One of the features reported in [4] was studied in more detail, showing correlation with the appearance of a growing $\mathrm{m}: \mathrm{n}=2: 1$ magnetic island at $4.7 \mathrm{~T}$ central magnetic field, in asymmetric configuration (see [4]) at line-integrated density of around $1 \times 10^{20} \mathrm{~m}^{-3}$. In Fig. 2 the spectrum of shot \#39848 is shown in correlation with a Mirnov Coil signal and an MHD frequency spectrum. Sudden bursts of emissions detected by CTS at frequencies multiple of the ion cyclotron frequency above and below the gyrotron frequency (at $0.8 \mathrm{GHz}$ in the spectrum) show a fast time evolution (at microsecond scale) which differs with respect to phenomena observed in $[1,2]$. Simultaneous variations of the stray radiation level in the vessel close to the launching port have been recorded.

New experiments were performed in 2016 at $4.7 \mathrm{~T}$ with two channels set to receive in parallel the two polarizations [6], both with frequency band enlarged to 4.2 $\mathrm{GHz}$ around the local oscillator frequency. In asymmetric configuration, emissions of another type described in [4] were observed and analyzed.

They are reported in Fig. 3, where the two channels of the receiver 1 (top, with chopping, detecting X-polarized wave), receiver 2 (without chopping, mostly O-wave) and Mirnov Coil signal are shown. Even if the appearance of these emissions is linked to the mode growth, the timing is not linked in phase with island rotation, and the duration is much less that the rotation period by at least one order of magnitude. 


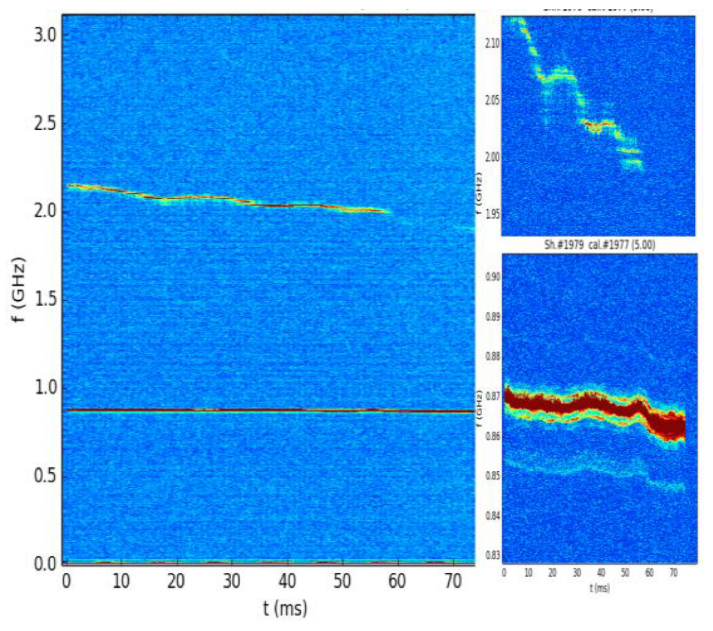

Fig. 1. Left: Spectrum obtained from the gyrotron stray power, with a pick-up at the EC launcher. The main gyrotron line which is stable in frequency at $0.9 \mathrm{GHz}$ from the Local Oscillator is accompanied by a spurious line variable in frequency around $1.2 \mathrm{GHz}$ above the gyrotron line. On the right a zoom in frequency of the two lines during the gyrotron pulse (the gyrotron line at the bottom)

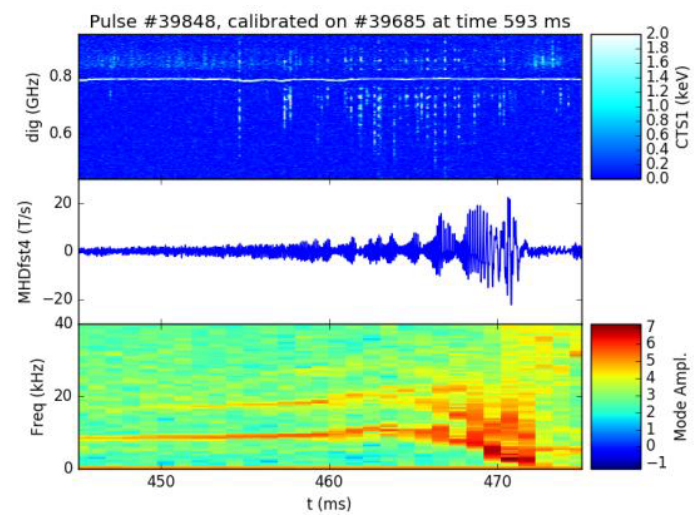

Fig. 2. Spectrum of shot $\# 39848$ is shown (top) in correlation with a Mirnov Coil signal (center) and MHD frequency spectrum (bottom). Bursts of emissions detected by CTS at frequencies multiple of the ion cyclotron frequency above and below the gyrotron frequency (at $0.8 \mathrm{GHz}$ in the spectrum)

\section{Diagnostic improvements}

In order to exclude in the spectrum signatures of the gyrotron stray (by comparison with the gyrotron spectrum in the same shot) and to verify the position of the emissions (comparing with the spectra received from a different line of sight), a new receiving line is being designed, that collects radiation from the plasma through a square corrugated antenna placed in the equatorial plane of the EC port n. 8, the same of the presently used EC and CTS antenna. A mirror steering mechanism is being designed to perform steering of the received beam from outside, without moving parts into the vessel, using the well-known Remote Steering waveguide properties, to have an independent viewing line that can intersect or not the probe beam. A switch will allow also a direct reception of the stray from the gyrotron line at the port level. The path of the receiving line combines reduced-section square corrugated waveguides, $88.9 \mathrm{~mm}$ circular corrugated waveguides or, in case of need, also a definitelength smooth circular waveguide and quasi-optical sections with mirrors instead of mitre bends.

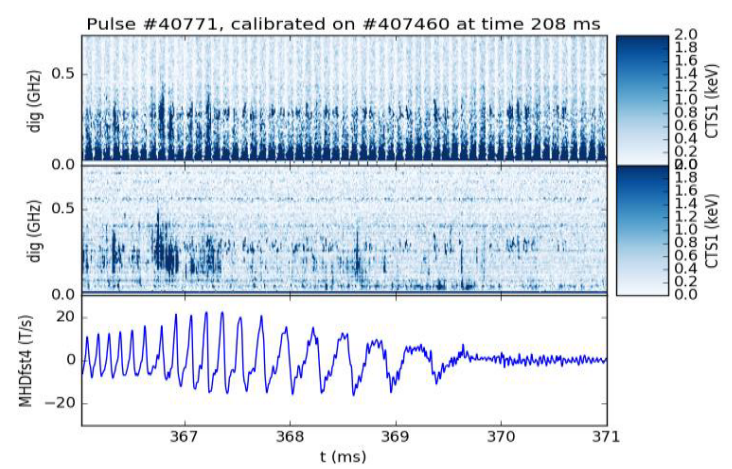

Fig. 3. Spectra of receiver 1 (top, with chopping, X-wave), receiver 2 (without chopping, O-wave) and Mirnov Coil signal for shot \#40771

\section{Acknowledgement}

This work has been carried out within the framework of the EUROfusion Consortium and has received funding from the Euratom research and training programme 2014-2018 under grant agreement No 633053. The views and opinions expressed herein do not necessarily reflect those of the European Commission.

\section{References}

1. Westerhof, E. et al. Strong Scattering of High Power Millimeter Waves in Tokamak Plasmas with Tearing Modes // Phys. Rev. Lett. 2009. V. 103, 125001.

2. Nielsen S.K. et al. Experimental characterization of anomalous strong scattering of mm-waves in TEXTOR plasmas with rotating islands // Plasma Phys. Control. Fusion 2013. V. 55,115003 .

3. Gusakov, E.Z., Popov, A.Yu. Theory of anomalous backscattering in second harmonic X-mode ECRH experiments // Phys. Plasmas 2016. V. 23, 082503.

4. Bruschi, A. et al., Observation of short time-scale spectral emissions at millimeter wavelengths with the new CTS diagnostic on the FTU tokamak // Nucl. Fusion 2017. V. 57, 076004.

5. Bin, W. et al., The upgraded Collective Thomson Scattering diagnostics of FTU // Fusion. Eng. Des. 2015. V. 96-97, P. 733-737.

6. Bin, W. et al., Advances in the FTU collective Thomson scattering system // Rev. Sci. Instrum. 2016. V. 87, 11E507. 